Revista Brasileira de Odontologia Legal - RBOL

\title{
Radiologia Forense
}

\section{IMPORTÂNCIA PERICIAL NA ANÁLISE DA RADIOPACIDADE DE RESINAS COMPOSTAS POR MEIO DE SISTEMA RADIOGRÁFICO DIGITAL.}

\section{Forensic importance in the analysis of composite radiopacity using digital radiograph system.}

\author{
Daphne Azambuja Hatschbach de AQUINO', Ângela FERNANDES², Luiz \\ Roberto Coutinho MANHÃES JUNIOR ${ }^{1}$.
}

1. Departamento de Odontologia, Radiologia Odontológica e Imaginologia, Curso de Odontologia, Faculdade de Odontologia e Medicina São Leopoldo Mandic, São Paulo, Brasil.

2. Departamento de Estomatologia, Radiologia Odontológica e Imaginologia, Curso de Odontologia, Universidade Federal do Paraná, Paraná, Brasil.

Informação sobre o artigo

Recebido em: 09 Jan 2017

Aceito em: 29 Jan 2017

\author{
Autor para correspondência \\ Luiz Roberto Coutinho Manhães Junior \\ Faculdade de Faculdade de Odontologia e Medicina São \\ Leopoldo Mandic - Radiologia Odontológica e \\ Imaginologia \\ Rua Dr. José Rocha Junqueira 13, Campinas, São Paulo, \\ Brasil. CEP: 13045-755 \\ E-mail: Irmanhaesir@hotmail.com.
}

\begin{abstract}
RESUMO
Introdução: A análise da radiopacidade nos exames radiográficos é importante ao cirurgião-dentista não apenas para a diferenciação entre estruturas dentais e material restaurador, mas também para verificação de infiltrações marginais e do diagnóstico de cáries secundárias ou recorrentes. Além de sua importância clínica, a diferenciação de materiais restauradores por sua radiopacidade apresenta potencial no âmbito forense, uma vez que pode auxiliar no processo de identificação humana. Objetivo: Este estudo teve como objetivo avaliar a radiopacidade das resinas compostas fotopolimerizáveis Tetric N Ceram, Z100, Z250 e Z350 por meio de radiografia digital. Métodos: Foi realizado um estudo experimental in vitro com 16 corpos de prova medindo $2 \times 4 \mathrm{~mm}$. Foi usada uma escala de alumínio com 9 degraus (com $1 \mathrm{~mm}$ de espessura cada) para comparação dos valores da escala dinâmica encontrados por meio das imagens digitais geradas no sistema digital radiográfico indireto. Foram feitas 12 leituras para cada resina avaliada e para cada um dos sete degraus da escala de alumínio em três datas diferentes. Para verificar qual dos degraus da escala de alumínio representaria os valores obtidos pelas resinas individualmente, os valores foram comparados com aqueles obtidos em cada degrau. Resultados: A resina Tetric mostrou maior similaridade com o degrau 1 da escala de alumínio, ou seja, o mais radiopaco. A resina Z100 apresentou valores próximos com o degrau 3. Os valores da resina Z250 apresentaram variabilidade entre os degraus 3 e 4, porém não mostrou correlação com os mesmos. A resina Z350 apresentou correlação muito boa, inversa e significativa com os degraus 3 e 4. Conclusão: Cada resina analisada apresentou características de radiopacidade singulares, que potencialmente viabilizam o auxilio a Odontologia Legal frente à necessidade de identificação humana. Em específico, a resina Tetric apresentou maior radiopacidade. Ainda assim, as resinas Z100, Z250 e Z350 apresentaram radiopacidade dentro dos padrões esperados (exigidos pela ISO 4049). Estudos futuros são necessários com outras resinas compostas para compreender melhor o potencial desta pesquisa para propósitos forenses.
\end{abstract}

PALAVRAS-CHAVE

Odontologia legal; Restauração dentária permanente; Radiografia dentária digital; Identificação humana. 


\section{INTRODUÇÃO}

A análise dos graus de radiopacidade das imagens radiográficas permite ao cirurgião-dentista a diferenciação entre estruturas dentais, material restaurador, lesão cariosa e qualidade do preenchimento do sistema de canais radiculares, distinguindo o material obturador da dentina e do osso alveolar ${ }^{1}$. As características das resinas compostas estão em constante transformação e muitas modificações estão sendo realizadas em sua matriz inorgânica a fim de aperfeiçoar esse material e torná-lo mais próximo do ideal. Dentre suas características destaca-se a radiopacidade (quantidade de níveis de cinza que compõem a imagem) que é muito importante quando se analisa a imagem radiográfica de um dente restaurado com resinas compostas. Cabe ressaltar que, apesar de ainda não haver um padrão de Densidade Óptica (DO) ideal estabelecido para os materiais restauradores, a radiopacidade mínima deve ser semelhante à do esmalte $^{2}$. A preocupação dos profissionais é a de que os materiais restauradores forneçam radiopacidade suficiente para ser visível no exame radiográfico, em conformidade com as normas da Associação Dental Americana ${ }^{3-5}$ e da International Organization for Standardization (ISO) 40496,7.

A radiografia digital emergiu nas últimas décadas como meio eficaz e rápido para a aquisição de imagens e armazenamento virtual. Além disto, o ambiente digital viabiliza a o ajuste de brilho, contraste, e outras características que permitem otimizar a qualidade da imagem e, portanto, contribuir para a visualização de estruturas anatômicas e patológicas $^{8-12}$. Além disso, como não emprega processamento químico, evita os erros inerentes a esta fase, reduz o tempo de exposição à radiação e diminui os riscos de efeitos deletérios aos pacientes ${ }^{13}$. A imagem digital pode ser obtida de duas formas: por meio de sensores eletrônicos ou ópticos sensíveis à radiação (CCD - Charge Coupled Device ou CMOS Complementary Metal Oxide Semiconductor) e por meio de placas de fósforo estimuladas (PSP - Phosphor Stimulate Plate).

Do ponto de vista pericial, tem-se no estudo da diferenciação de materiais restauradores um mecanismo em potencial para o auxílio do processo de identificação humana em Odontologia Legal, não somente pelo potencial discriminatório destes materiais pela sua radiopacidade, mas também pela possibilidade de distinguir o material dos tecidos dentais adjacentes ${ }^{7}$. Em especial, a investigação acerca da radiopacidade de resinas compostas tem um papel significante no atual cenário Odontológico mundial, uma vez que restaurações estéticas substituem cada vez mais restaurações de amálgama na rotina clínica $^{14}$. A vasta produção de materiais estéticos em Odontologia originou uma grande variação nos níveis de radiopacidade entre restaurações, o que permitiu sua investigação e até mesmo potencial utilidade forense. Neste contexto, o presente estudo avaliou a importância pericial na análise da radiopacidade de 4 resinas compostas fotopolimerizáveis por meio de um sistema de radiografia digital. 


\section{MATERIAIS E MÉTODOS}

Este estudo experimental in vitro direcionado teve metodologia baseada no trabalho de Rizon et al. $(2010)^{15}$, porém sua amostra foi modificado, consistindo de 16 corpos de prova medindo $2 \mathrm{~mm}$ de espessura por $4 \mathrm{~mm}$ de diâmetro cada. Estes corpos de prova foram elaborados utilizando-se as seguintes resinas compostas: Tetric N Ceram (Ivoclar Vivadent, Barueri, São Paulo, Brasil), Z100A2, Z250 XT-OA2 e Z350 XT-A2 (3M Company, St. Paul, Minnesota, USA). Além das resinas compostas, utilizou-se como mecanismo controle uma escala de alumínio de 9 degraus com espessura gradual de 1 $\mathrm{mm}$ cada. As resinas compostas foram fotopolimerizadas utilizando-se um aparelho Demetron LC (Kerr Corporation, California, USA). As imagens radiográficas foram obtidas utilizando um aparelho radiômetro digital (Newdent Equipamentos, Ribeirão Preto, São Paulo, Brasil); um sistema radiográfico digital Express (Instrumentarium, Tuusula, Finlândia); um aparelho de raios $X$ intrabucal de alta frequência Focus (Instrumentarium, Tuusula, Finlândia) e um scanner de processamento conectado a um computador com o software Cliniview (Instrumentarium, Tuusula, Finlândia). As imagens foram processadas pelo programa Image $\mathrm{J}$ (National Institute of Health Imaging, Mariland, EUA).

De acordo com Rizon et al. (2010) ${ }^{15}$, foram confeccionadas 4 placas de acrílico transparentes medindo $5,7 \times 7,1 \mathrm{~cm}$ segundo. Cada placa foi dividida em 4 quadrantes e no centro de cada quadrante foram feitos orifícios de $4 \mathrm{~mm}$ de diâmetro, cuja profundidade correspondeu à espessura total da placa, ou seja, de 2mm (Figura 1). Estes orifícios corresponderam de sítios para a inserção e compactação das resinas compostas pesquisadas. Cada placa de acrílico foi utilizada para um tipo de resina composta. As resinas compostas foram identificadas em cada placa com marcação permanente.

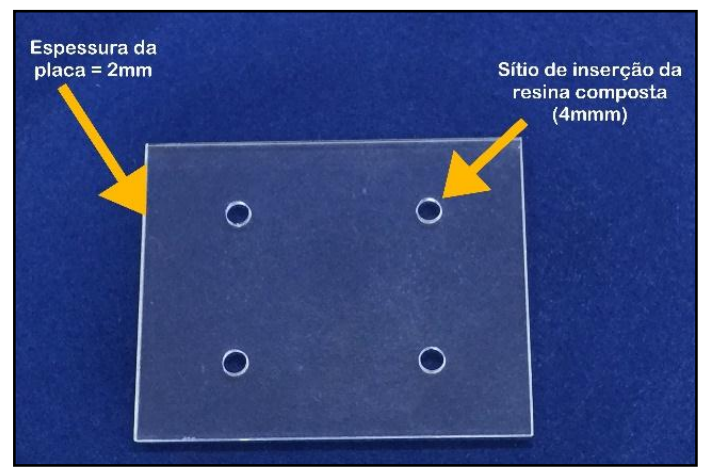

Figura 1 - Placa de acrílico com 4 sítios de inserção para resinas compostas.

As resinas foram inseridas e compactadas individualmente nos orifícios das placas, com o uso de uma espátula de resina para evitar bolhas ou má adaptação. A compactação do material ocorreu até ser obtida a homogeneidade no preenchimento total do orifício da placa. Para garantir lisura adequada e espessura dos corpos de prova, cada placa de acrílico foi fixada entre 2 placas de vidro com $6 \mathrm{~mm}$ de espessura e então foram comprimidas manualmente. Após a compressão, as resinas foram polimeralizadas com aparelho fotopolimerizador (Demetron LC, Kerr Corporation, California, USA) durante 40 segundos e com $440 \mathrm{~mW} / \mathrm{cm} 2$ de intensidade, com a ponta do fotopolimerizador posicionada verticalmente em relação ao corpo de prova. Antes da polimerização de cada resina, a intensidade 
da luz do fotopolimerizador foi aferida com aparelho Radiômetro digital (Newdent Equipamentos, Ribeirão Preto/SP/Brasil). Os procedimentos foram repetidos para todos os corpos de prova, já que foram confeccionados individualmente, sendo mantida a mesma sequência para cada um. Então, os corpos de prova foram divididos em 4 grupos conforme o tipo de resina composta analisada:

- G100 - grupo dos corpos de prova gerados com a resina composta fotopolimerizável Z100;

- G250 - grupo dos corpos de prova gerados com a resina composta fotopolimerizável Z250;

- G350 - grupo dos corpos de prova gerados com a resina composta fotopolimerizável Z350;

- GT - grupo dos corpos de prova gerados com a resina composta fotopolimerizável Tetric N Ceran.

A escala de alumínio foi posicionada de forma padronizada entre dois orifícios de cada placa, sobre o sensor radiográfico. Foram obtidas duas radiografias de cada placa de acrílico, juntamente com a escala de alumínio para que a radiopacidade das resinas pudesse ser avaliada (Figura 2).

Para padronização do experimento, a escala de alumínio foi posicionada entre dois corpos de prova, mantendo-os alinhados na altura do degrau 4. Estabeleceu-se o degrau 1 (mais radiopaco) na parte inferior da radiografia e o degrau 7 (mais radiolúcido) na parte superior.

As radiografias foram realizadas com - sistema radiográfico digital indireto Express (Instrumentarium, Tuusula,
Finlândia). As PSP foram colocadas em um suporte, e sobre as mesmas foram colocadas as placas de acrílico com os corpos de prova. Foi utilizado o aparelho de raios $X$ intraoral de alta frequência da marca Focus (Instrumentarium, Tuusula, Finlândia) com o feixe central dos raios $\mathrm{x}$ incidindo em ângulo vertical de $90^{\circ} \mathrm{com}$ a superfície do sensor, utilizando $70 \mathrm{kV}$ e $10 \mathrm{~mA}$, a uma distância de $30 \mathrm{~cm}$ foco-placa com 0,1 segundo de tempo de exposição. Após a exposição aos raios $\mathrm{X}$, as imagens latentes foram levadas para o scanner de processamento, conectado a um computador para que, no software Cliniview (Instrumentarium, Tuusula, Finlândia) fosse feita a leitura com padrão de brilho em 50 , contraste em 0 e gama em 10. As imagens obtidas foram exportadas no formato JPG (Joint Photographic Groups) de forma que ficassem disponíveis ao programa Image J (National Institute of Health Imaging, Mariland, EUA).

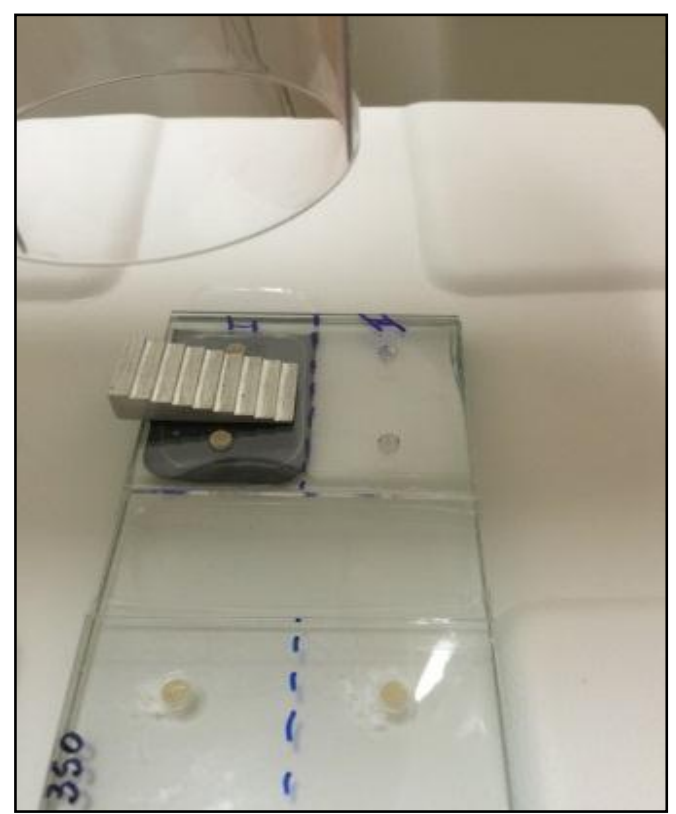

Figura 2 - Placa de acrílico e escala de alumínio posicionadas sobre a placa de fósforo para obtenção da radiografia. 
Para coletar os dados de radiopacidade de cada uma das imagens dos corpos de prova, era marcada uma área quadrada de aproximadamente $3 \mathrm{~mm}$, posicionada ao centro de cada imagem do corpo de prova do lado esquerdo e do lado direito de cada radiografia, respectivamente. $\mathrm{Na}$ sequência, eram obtidos os valores da radiopacidade de cada um dos degraus da escala de alumínio de cada radiografia para posterior comparação. Esse procedimento foi realizado em 3 datas diferentes, com intervalo de 15 dias entre as leituras, com o intuito de minimizar erros de leitura. Sendo assim, foram obtidos 96 dados das 3 leituras de cada corpo de prova, os quais foram tabulados de acordo com os grupos e submetidos à análise estatística.

Foram realizadas análises descritivas e testes inferenciais com significância de $95,0 \%$. A distribuição dos dados e as variâncias foram analisadas pelos testes de Shapiro-Wilk e Bartlett, respectivamente. O teste de correlação intraclasse (ICC) foi utilizado para avaliar a reprodutibilidade intra-avaliador. A comparação entre os valores de leitura (médias em tons de cinza) das resinas e dos degraus da escala de alumínio foi feita pelo teste de KruskalWallis. O teste de correlação de Spearman (rS) observou a relação entre os valores de leitura da escala dinâmica das resinas e dos degraus da escala de alumínio mais próximos. Os softwares utilizados para as análises foram o GraphPad 6.0 (GraphPad Software, California, USA) e o BioEstat 5.0 (Instituto Mamiraua, Tefé, Amazonas, Brasil), sendo que o nível de significância foi acertado para $5 \%$.

\section{RESULTADOS}

Ao total, 12 leituras foram realizadas para cada resina composta e para cada degrau da escala de alumínio, nas 3 datas diferentes.

O teste ICC revelou excelente reprodutibilidade intra-avaliador (ICC $=0.9972, p<0.0001)$.

As diferenças de radiopacidade entre cada grupo são apresentadas na Figura 3.

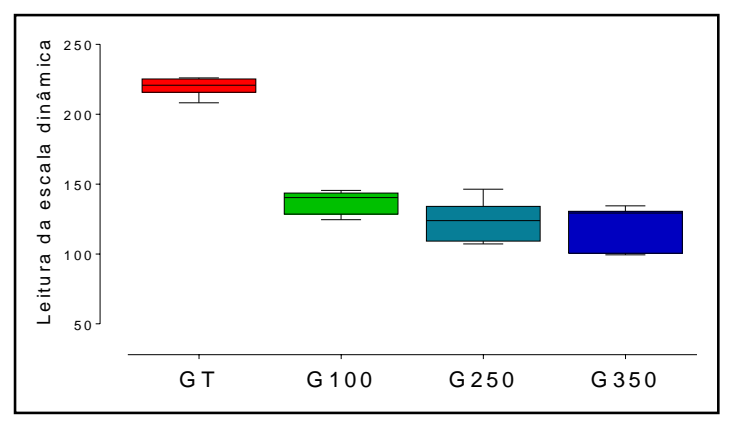

Figura 3 - Valores de leitura das médias em tons de cinza (leitura da escala dinâmica) em função dos grupos amostrais. GT - grupo dos corpos de prova gerados com a resina composta fotopolimerizável Tetric N Ceran; G100 - grupo dos corpos de prova gerados com a resina composta fotopolimerizável Z100; G250 - grupo dos corpos de prova gerados com a resina composta fotopolimerizável Z250; G350 - grupo dos corpos de prova gerados com a resina composta fotopolimerizável Z350.

Diferenças estatisticamente significantes foram observadas entre os grupos GT e G100 ( $p=0.0213$ ), GT e G250 $(p<0.0001)$ e GT e G350 ( $p<0.0001)$ (teste de Kruskal-Wallis). Não foram observadas diferenças estatisticamente significantes entre os grupos G100, G250 e G350 $(p>0.05)$.

Para verificar qual dos degraus da escala de alumínio representaria o conjunto de valores obtidos pelas resinas 
individualmente, os valores foram comparados com aqueles obtidos em cada um desses degraus, como mostram as Figuras de 4 a 7 .

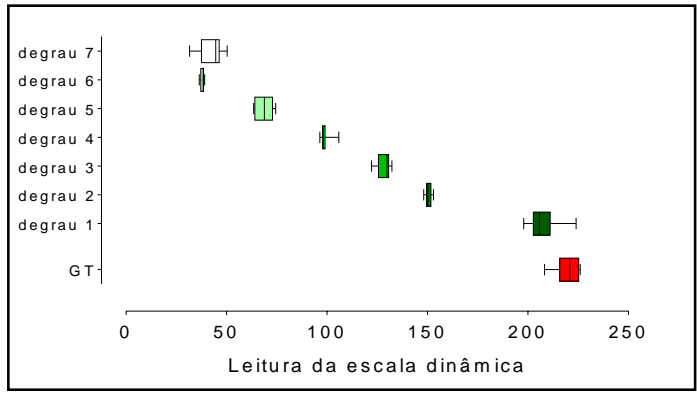

Figura 4 - Valores de leitura das médias em tons de cinza (leitura da escala dinâmica) em função da resina Tetric (GT) e dos degraus da escala de alumínio.

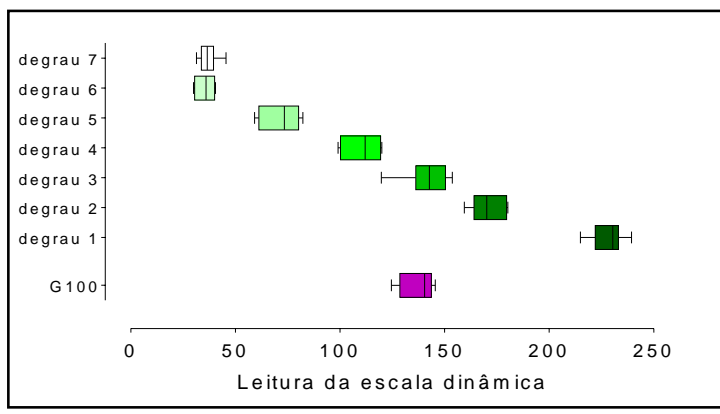

Figura 5 - Valores de leitura das médias em tons de cinza (leitura da escala dinâmica) em função da resina $Z 100$ e dos degraus da escala de alumínio.

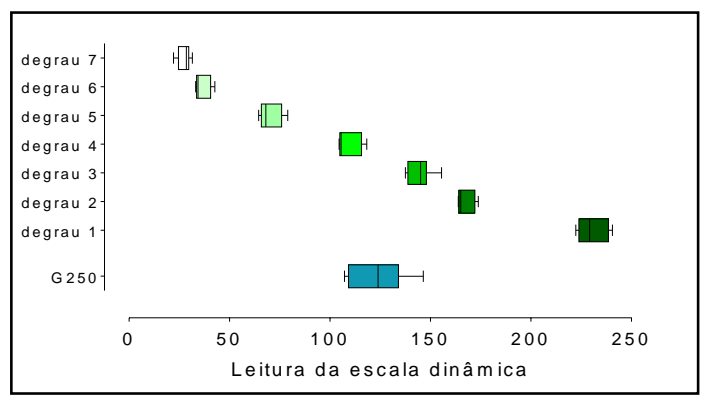

Figura 6 - Valores de leitura das médias em tons de cinza (leitura da escala dinâmica) em função da resina $Z 250$ e dos degraus da escala de alumínio.

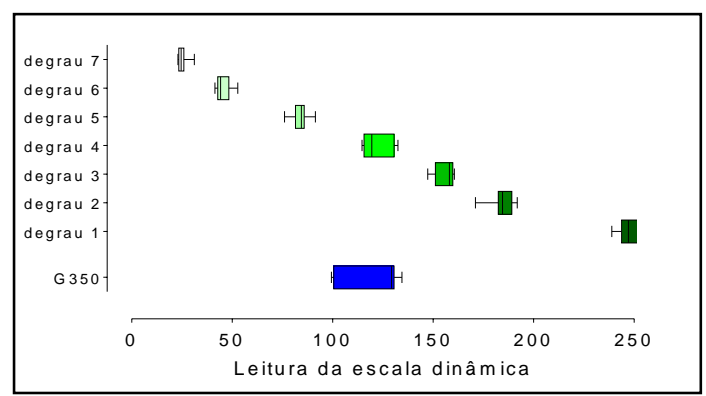

Figura 7 - Valores de leitura das médias em tons de cinza (leitura da escala dinâmica) em função da resina $Z 350$ e dos degraus da escala de alumínio.

O grupo GT mostrou maior similaridade com o degrau 1 da escala de alumínio, não apresentando diferenças estatisticamente significantes (KruskalWallis, $p=0.3713$ ) entre os valores da leitura entre ambos. Todos os demais degraus apresentam diferenças estatisticamente significantes $(p<0.05)$ com a resina, indicando que não seriam bons representantes dessa resina.

Já para o grupo G100, embora o degrau $3(p=0.8346)$ seja aquele com valores mais próximos dos valores de G100, os degraus $2(p=0.0905)$ e $4(p=0.1438)$ também não apresentam diferenças estatisticamente significativas com os valores observados para a resina. Os valores obtidos para os demais degraus mostraram diferenças estatisticamente significantes $(p<0.05)$ com aqueles da resina.

Da mesma forma, devido à variabilidade observada nos valores do grupo G250, este se situou melhor representado entre os degraus $3(p=0.3154)$ e $4(p=0.2470)$, sendo que a diferença com os demais degraus foi significante $(p<0.05)$.

O grupo G350 também apresentou grande variabilidade e os valores se 
situaram mais próximos aos do degrau 4 $(p=0.9299)$, embora também não tenham sido observadas diferenças estatisticamente significantes com os degraus 3 ( $p=0.1038)$ e $5(p=0.1239)$. Com os demais degraus houve diferenças estatisticamente significantes $(p<0.05)$.

O teste de correlação de Spearman (rS) entre os valores de leitura da escala dinâmica das resinas e dos degraus da escala de alumínio mais próximos revelou que não houve correlação entre o grupo GT e o degrau 7 ( $r S=0.18, p=0.5717)$. Da mesma forma, o grupo G100 não apresentou correlação com os degraus 2 ( $r S=0.45, p=0.1376), 3$ ( $r S=0.34, p=0.2756$ ) ou 4 ( $r S=0.43, p=0.1591)$. O grupo $G 250$ não mostrou correlação com os degraus 3 $(r S=0.48, \quad p=0.1110) \quad$ ou $4 \quad(r S=0.53$, $\mathrm{p}=0.0750$ ). Entretanto, o $\mathrm{G} 350$ apresentou correlação muito boa, inversa e significativa com os degraus $3(r S=-0.78, p=0.0030)$ e 4 $(r S=-0.71, p=0.0102)$, indicando que quanto maior era o valor observado para a resina Z350, menor era o valor da escala observada tanto para o degrau 3 quanto 4 .

Não houve, entretanto, nenhuma outra relação entre os valores oriundos dos grupos GT, G100 e G250 com nenhum degrau da escala de alumínio. Assim, de uma maneira geral, a correspondência entre os valores de leitura da escala dinâmica de resinas e da escala de alumínio não são intercambiáveis.

\section{DISCUSSÃO}

Neste estudo optou-se pela análise da radiopacidade das resinas Tetric, Z100, Z250 e Z350 por serem bastante utilizadas pelos cirurgiões-dentistas brasileiros. Desta forma, os achados obtidos poderiam não somente auxiliar a identificação humana propriamente dita, mas também ser principalmente útil para a casuística da perícia nacional.

A confiabilidade do presente estudo foi assegurada considerando-se medidas tidas como controle de qualidade metodológica. Primeiramente pela realização de três leituras dos 16 corpos de prova em dias diferentes, seguida pelo teste de correlação intraclasse (demonstrando reprodutibilidade excelente), e pela seleção de uma ferramenta para análise de imagens tida como confiável pela literatura relacionada $^{16,17}$.

Para verificar qual dos degraus da escala de alumínio representaria o conjunto de valores obtido pelas resinas compostas individualmente, foram comparados os valores obtidos pela leitura da radiopacidade da resina e os valores obtidos em cada um dos degraus. Foi observado que os valores da resina Tetric apresentaram diferenças significantes quando comparados com os valores das resinas Z100, Z250 e Z350. Além disso, a Tetric foi a resina que apresentou valores de radiopacidade mais próximos do degrau 1 (o mais radiopaco da escala de alumínio). Sendo assim, no momento de se escolher uma resina composta, deve-se considerar o material restaurador com radiopacidade similar ou ligeiramente acima daquela do esmalte $^{18}$, pois este pode aperfeiçoar a visualização de lesões de cárie por meio de radiografias.

A resina Z100 apresentou valores mais próximos com o degrau 3 da escala de alumínio. Os valores da resina Z250 
apresentaram variabilidade e esta se situou entre os degraus 3 e 4, porém não mostrou correlação com os mesmos. A resina Z350 apresentou correlação muito boa, inversa e significativa com os degraus 3 e 4 que correspondem à espessura de 3 e $4 \mathrm{~mm}$ de alumínio, respectivamente. Segundo a ISO 4049 a densidade radiográfica da dentina apresenta radiopacidade semelhante à do alumínio, na proporção de 1:1. Resultados similares foram encontrados por Ermis et al. $(2014)^{16}$ que avaliaram a radiopacidade de 10 tipos de resinas compostas (entre elas a Z250) e compararam com os valores de dentina e esmalte humanos. Todas as resinas compostas investigadas apresentaram valores diferentes de radiopacidade, mas maiores que a dentina.

$$
\text { Almeida e Teixeira }(2009)^{19}
$$

verificaram a radiopacidade de diferentes resinas e ionômeros de vidro por meio de radiografia digital. Utilizaram metodologia similar à deste estudo, inclusive uma escala de alumínio, um espécime de amálgama e um de chumbo. Os resultados demonstraram que as resinas Z250 e NL apresentaram valores de radiopacidade superiores ao valor mínimo recomendado pela Associação Dental Americana, que é de $4 \mathrm{~mm}$ de espessura de alumínio. Já as resinas Z350 e BRI, assim como os cimentos de ionômero de vidro, apresentaram valores inferiores.

Considerando a metodologia, para as exposições radiográficas foram usados neste estudo, uma placa de fósforo de armazenamento e um equipamento de raios $X$ odontológico com regime de exposição de 70 kVp e 10 mA. A distância foco-objeto foi de $30 \mathrm{~cm}$, e o tempo de exposição foi de 0,1 segundo. Ao passo que Tarcin et al. $(2016)^{20}$ fizeram uso de uma placa de fósforo de armazenamento e um equipamento de raios $X$ odontológico com $70 \mathrm{kVp}$ e $8 \mathrm{~mA}$, com tempo de exposição de 0,2 segundo. Enquanto Almeida \& Teixeira $(2009)^{19}$ utilizaram um tempo de 0,3 segundos. Porém, segundo Sabbagh et al. $(2004)^{21}$ e Tsuge et al. (2009) $)^{22}$ o tempo de exposição não interfere na radiopacidade da imagem, quando considerados pequenos intervalos de tempo.

Oztas et al. (2012) ${ }^{23}$ afirmaram que a Z250 foi mais radiopaca que a dentina no sistema digital. Enquanto para Flores et al. $(2013)^{24}$ a resina Z250 demonstrou ser mais radiopaca que o esmalte. No estudo de Dukic et al. (2012) ${ }^{25}$ a Z250 foi classificada em $14^{\circ}$ lugar de radiopacidade, entre as 30 resinas estudadas por aqueles autores. Apesar da controvérsia em relação ao grau de radiopacidade da resina Z250, tanto neste estudo como nos demais anteriormente citados, sua radiopacidade apresentou-se dentro dos padrões estabelecidos internacionalmente.

Ao se fazer a seleção de um material restaurador para uso clínico, várias características do material devem ser avaliadas em conjunto. Porém, se for considerar a radiopacidade isoladamente, observou-se que a resina Tetric foi a que demonstrou o maior valor. Entretanto, as resinas Z100, Z250 e Z350 apresentaram radiopacidade dentro dos padrões estabelecidos pela ISO $4049^{6}$.

Já do ponto de vista pericial, o presente trabalho contribui significativamente traçando um perfil da radiopacidade observada nos quatro 
materiais restauradores analisados. Em Odontologia Legal, o processo de identificação humana é realizado em formato comparativo ${ }^{26}$. Diferentemente dos demais meios de identificação humana, como a Papiloscopia e a análise de padrões genéticos (DNA), a Odontologia não requer um número mínimo de pontos concordantes para estabelecer uma identificação positiva $^{27}$. Contudo, diversas são as características dentais e maxilofaciais que fornecem potencial discriminatório para identificação. Porém, as restaurações dentais registradas por meio de radiografias figuram entre os achados mais utilizados no processo de identificação. Apesar da tendência ao regresso nos tratamentos restauradores em vista da crescente demanda por tratamentos preventivos em Odontologia, a prevalência de dentes restaurados ainda é alta, especialmente em países em desenvolvimento como o Brasil, fazendo com que restaurações sejam constantes fontes de evidência forenses para identificação humana. A primeira contribuição deste estudo ao meio forense é assegurar que a radiopacidade das resinas compostas utilizadas esteja dentro dos padrões recomendados em controles de qualidade. Desta forma, faz-se possível a visualização das mesmas em exames radiográficos periciais. Assim, exames periciais mais confiáveis são alcançados uma vez que as resinas são visualizadas adequadamente.

Mais adiante, o presente estudo adentra de fato na contribuição do estudo da radiopacidade de resinas compostas para identificação humana quantificando resultados individuais para cada resina analisada. Estudos similares foram realizados com objetivos forenses, contudo foi investigada contribuição da morfologia das restaurações dentais e não a sua radiopacidade 28,29 . Contudo, um estudo de cunho forense ${ }^{30}$ se destaca na literatura por apresentar resultados que limitam 0 potencial da diferenciação entre a radiopacidade de materiais restauradores para identificação humana. Wenzel et al. $(1998)^{30}$ demonstraram que materiais restauradores podem não ser diferenciados entre si por sua radiopacidade. Apesar dos resultados opostos ao presente estudo, os autores utilizaram metodologia diferente, incluindo dentes humanos extraídos, e 8 materiais restauradores, dos quais apenas 2 eram resinas compostas. No campo da tomografia computadorizada (TC), a metodologia é ainda mais diferenciada, na qual a "radiopacidade" dos materiais restauradores é comparada quantificando-a em Hounsfield Units ${ }^{31}$. Da mesma forma, estudos em TC acusam o potencial desta abordagem para identificação humana. Contudo, os resultados reportados na literatura são, ainda hoje, incipientes. Espera-se que no futuro o odontolegista tenha em seu arsenal uma ferramenta adicional que permitirá discriminar com precisão qual a resina (ou as resinas incluídas em um intervalo de radiopacidade) foi utilizada para restaurar os dentes de uma vítima não identificada.

Uma aplicação adicional desta abordagem para o meio forense se dá na esfera administrativa, na qual auditor odontológico poderia detectar por radiografias falhas no tratamento, como a utilização de resinas compostas para dentes 
anteriores em dentes posteriores. Como consequência poderia $\circ$ procedimento poderia ser glosado. Ainda do ponto de vista forense, recomenda-se que o Cirurgiãodentista clínico atue em conformidade com o Código de Ética Odontológica ${ }^{32}$, preenchendo corretamente $\mathrm{o}$ prontuário odontológico (e atualizando), incluindo informações não apenas acerca do procedimento executado, mas também da marca e tipo de resina utilizada. Visando, desta forma, contribuir para o processo de identificação humana.

Estudos futuros são necessários para dar continuidade a esta pesquisa no âmbito forense. Registros odontológicos obtidos em perícias de identificação humana poderão ser estudados com a metodologia proposta, uma vez que o material restaurador seja de procedência conhecida. Desta forma, limitações inerentes da metodologia presente poderão ser evitadas, como o ensaio in vitro que excluí a comparação direta entre a radiopacidade do material restaurador e do dente humano. Assim como muitos outros materiais restauradores deverão ser incluídos para o estudo de sua radiopacidade.

\section{CONCLUSÃO}

Cada uma das resinas estudadas apresentou nível de radiopacidade distinto, todos estes dentro dos padrões de controle de qualidade. Contudo, sua aplicação no meio forense carece de mais estudos similares (uma vez que os resultados apresentados são incipientes) incluindo diferentes tipos de resinas e ensaios utilizando dentes extraídos. Apesar de escassa, a literatura que cerceia o presente estudo indica um potencial a ser explorado. Em confirmação, os resultados hora apresentados poderão futuramente beneficiar a Odontologia Legal, principalmente em suas esferas criminal e administrativa.

\section{ABSTRACT}

Introduction: The analysis of radiopacity in radiographic exams is important for dentists not only for the differentiation between dental structures, but also for the assessment of infiltration in dental restorations and the diagnosis of dental caries. Besides the clinical significance, the differentiation of restorative materials based on their radiopacity is potentially useful in Forensic Odontology because it may support the human identification process. Objective: This study aimed to assess the radiopacity of the composites Tetric N Ceram, Z100, Z250 and Z350 through digital radiographs. Methods: An experimental in vitro study was performed with 16 test samples measuring $2 \times 4 \mathrm{~mm}$. An aluminum scale with 9 levels (thickness of $1 \mathrm{~mm}$ for each level) was used as a comparative source in face of the radiopacity levels detected for the composites in the digital radiograph system. The radiopacity of each composite and each level of the scale was analyzed 12 times, in 3 different moments. The radiopacity values obtained between the scale and the composites were statistically associated to indicate which scale level represents better the composite analyzed. Results: The composite Tetric corresponded to the scale level 1 (the most radiopaque). The composite Z100 presented values closer to the scale level 3. The composite Z250 ranged between levels 3 and 4, but did not presented statistical association. The composite Z350 presented statistical association with scale levels 3 and 4 . Conclusion: Each composite presented distinct characteristics of radiopacity that could possibly contribute to the human identification process in Forensic Odontology. In specific, the composite Tetric had the highest radiopacity value. Despite less radiopaque, the composites Z100, Z250 and Z350 presented values within the standards expected (required by ISO 4049). Future studies are necessary with other composites in order to understand better the potential application for forensic purposes.

\section{KEYWORDS}

Forensic dentistry; Permanent dental restoration; Digital dental radiography; Human identification. 


\section{REFERÊNCIAS}

1. Carvalho Filho IB, Pontual MLA, Khoury HJ, Pontual AA, Devito KL, Silveira MMF. Influência do tempo de exposição e da kVp na radiopacidade de cimentos endodônticos. RFO. 2008; 13(2): 39-44. http://dx.doi.org/10.5335/rfo.v13i2.625.

2. Pereira ASR, Pires MM, Figueiredo JAP, Mota EG, Pires LAG. Análise dos níveis de cinza de 4 resinas compostas microhíbridas utilizando um sistema de radiografia digital direto. Rev Odonto Ciência. 2005; 20(47): 3-10.

3. American Dental Association. Council on Dental Materials, Instruments and Equipment. The desirability of using radiopaque plastic in dentistry: a status report. J Am Dent Assoc. 1981; 102(3): 347-9.

http://dx.doi.org/10.14219/jada.archive.198 $\underline{1.0058}$.

4. American Dental Association. Council on Dental Materials, Instruments and Equipment. Status report on posterior composites. J Am Dent Assoc. 1983; 107(1): $\quad$ 74-6. http://dx.doi.org/10.14219/jada.archive.198 3.0169.

5. American Dental Association. Council on Dental Materials and Equipment. Obstacles to the development of a standard for posterior composite resins. J Am Dent Assoc. 1989; 118(5): 649-51. http://dx.doi.org/10.14219/jada.archive.198 $\underline{9.0070 .}$

6. ISO. International Standard 4049:2000. Polymer based fillings, restorative and luting materials. Geneva: International Organization for Standardization Dentistry, 2009.

7. Corte-Real A, Veira DN. Identificação em medicina dental forense. Portugal: Coimbra; 2015.

8. Botelho TL, Mendonça EF, Cardoso LLM. Contribuição da radiografia digital na clínica odontológica. Robrac. 2003; 12(33): 55-9.

9. Coclete GA, Tavano O, Pavan AJ. Comparação das densidades ótica e radiográfica, analisadas pelo fotodensiômetro MRA e pelo sistema digital Digora. Rev Odontol UNESP. 2003; 32(1): 93-8.
10. Kreich EM, Leal GA, Slusarz PAA, Santini RM. Imagem digital na Odontologia. Ciênc Biol Saúde. 2005; 11(3/4): 53-61. http://dx.doi.org/10.5212/publicatio\%20uep g.v11i3.397.

11. Pasquali AAG, Matson MR, Raitz R. Estudo comparativo da densidade radiográfica de cimentos resinosos. Rev Odontol Univ São Paulo. 2009; 21(3): 23943.

12. Bicheri SAV, Victorino FR. Comparative evaluation of radiopacity of MTA Fillapex ${ }^{\circledR}$ endodontic sealer through a digital radiograph system. RSBO. 2013; 10(2): 149-52.

13. Cruz GA, Morais LC, Médici Filho E, Castilho JCM. Utilização de radiografia digital em Odontologia. Rev ABO Nac. 2004: 12(5): 283-6.

14. Hemasathya BA, Balagopal S. Study of composite restorations as a tool in forensic identification. J Forensic Dent Sci. 2013; 5(1): 35-41. http://dx.doi.org/10.4103/09751475.114561.

15. Rizon P, Visentin SC, Larentis N, Hehn L, Fontanella VRC. Avaliação da densidade óptica de três resinas compostas fotopolimerizáveis associadas à estrutura dental. Stomatos. 2010; 16(31): 77-86.

16. Ermis RB, Yildirim D, Yildiz G, Gormez O. Radiopacity evaluation of contemporary resin composites by digitization of images. Eur J Dent. 2014; 8: 342-7. http://dx.doi.org/10.4103/13057456.137644.

17. Kapila R, Matsuda Y, Araki K, Okano T, Nishikawa K, Sano T. Radiopacity measurement of restorative resins using film and three digital systems for comparison with ISO 4049: International Standard. Bull Tokyo Coll. 2015; 56(4): 207-14.

http://dx.doi.org/10.2209/tdcpublication.56. $\underline{207}$.

18. Turgut MD, Attar N, Onen A. Radiopacity of direct esthetic restorative materials. Oper Dent. 2003; 28(5): 508-14.

19. Almeida FA, Teixeira HM. Radiopacidade das Resinas Compostas. XVII Congresso de Iniciação Científica I Congresso de Iniciação em Desenvolvimento Tecnológico e Inovação. UFPE. 2009.

20. Tarcin B, Gumru B, Peker S, Overcoglu HS. Evaluation of radiopacity of bulk-fill flowable composites using digital 
radiography. Oper Dent. 2016; 41(4): 42431. http://dx.doi.org/10.2341/15-153-L.

21. Sabbagh J, Vreven J, Leloup G. Radiopacity of resin-based materials measured in film radiographs and storage phosphor plate (Digora). Oper Dent. 2004; 29(6): 677-84.

22. Tsuge T. Radiopacity of conventional, resin-modified glass ionomer, and resinbased luting materials. J Oral Sci. 2009; 51(2): 223-30. http://dx.doi.org/10.2334/josnusd.51.223.

23. Oztas B, Kursun S, Dinc G, Kamburoglu K. Radiopacity evaluation of composite restorative resins and bonding agents using digital and film $\mathrm{x}$-ray systems. Eur $\mathrm{J}$ Dent. 2012; 6: 115-22.

24. Flores ME, Halter Neto F, Boscolo FN, Royani G, Damian MF. Radiopacity assessment of composite resins through digital systems and InSight intraoral film. RFO. 2013; 18(2): 175-9. http://dx.doi.org/10.5335/rfo.v18i2.2563.

25. Dukic W, Delija B, Derossi D, Dadic I. Radiopacity of composite dental materials using a digital X-ray system. Dent Mater J. 2012; $\quad 31(1)$ : $\quad$ 47-53. http://dx.doi.org/10.4012/dmj.2011-119.

26. Silva RF, Franco A, Souza JB, Picoli FF, Mendes SD, Nunes FG. Human identification through the analysis of smile photographs. Am J Forensic Med Pathol. 2015; 36(2): 71-4. http://dx.doi.org/10.1097/PAF.0000000000 000148.

27. Acharya $A B$, Taylor JA. Are a minimum number of concordant matches needed to establish identity in forensic odontology? J Forensic Odontostomatol. 2003; 21(1): 613.

28. Zondag $\mathrm{H}$, Phillips VM. The discrimination potential of radio-opaque composite restorations for identification: Part 3. J Forensic Odontostomatol. 2009; 27(1): 2732.

29. Hemasathya BA, Balagopal S. A study of composite restorations as a tool in forensic identification. J Forensic Dent Sci. 2013; 5(1): 35-41. http://dx.doi.org/10.4103/09751475.114561.

30. Wenzel A, Hintze $H$, Horsted-Bindslev P. Discrimination between restorative dental materials by their radiopacity measured in film radiographs and digital images. J Forensic Odontostomatol. 1998; 16(1): 813.

31. Jackowski C, Lussi A, Classens $M$, Kilchoer T, Bolliger S, Aghayev E et al. Extended CT scale overcomes restoration caused streak artifacts for dental identification in CT-3D color encoded automatic discrimination of dental restorations. J Comp Assist Tomogr. 2006; 30(3): 510-3.

32. Brasil. Conselho Federal de Odontologia. Resolução CFO 118. Revoga o Código de Ética Odontológica aprovado pela Resolução CFO-42/2003 e aprova outro em substituição. Disponível em: http://cfo.org.br/wpcontent/uploads/2009/09/codigo etica.pdf. Acesso em: 20 de janeiro de 2017. 